\title{
The Influence of Trait Empathy on Implicit Altruism
}

\author{
Xie $\mathrm{Ma}^{1}$, Danna Luo ${ }^{1-}$, Ting Deng ${ }^{2}$, Yun Tao* \\ 1,1-2, ${ }^{2}$ Department of Education, Yunnan Normal University Key Laboratory of EducationalInformation for \\ Nationalities, Yunnan Normal University \\ ${ }^{1}$ These authors contributed equally to this work and should be considered co-first authors \\ *Corresponding author. Email: taoyun2011@126.com
}

\begin{abstract}
The influence of empathy on personal altruism is a hot topic in international research in recent years. Through combing the previous studies on empathy and altruism, it is found that those studies get contradictory results. Therefore, in this study, implicit association text were used to explore whether there are differences in implicit altruism among people with different empathy abilities. According to the average score of Interpersonal Reactivity Index, 67 subjects were divided into high empathy group and low empathy group. The accuracy, response time and IAT effect (response time of incompatible task less response time of compatible task ) of the implicit altruism experiment were compared between the two groups. The results showed that compared with the incompatible tasks, the compatible task had higher accuracy and shorter response time. In addition, the IAT effect of the high empathy group was significantly greater than that of the low empathy group, indicating that the higher the empathy was, the more automatic the altruistic tendency was. This study provides some supporting evidence for the promotion of altruistic behavior by empathic education.
\end{abstract}

Keywords: empathy, altruism, implicit association experiment

\section{INTRODUCTION}

Altruism refers to a behavior that people sacrifice their own interests for the benefit of others [1], such as donating money, donating blood, volunteering, etc, which is of great significance for individuals to maintain interpersonal relations, promote social unity and progress. Influencing factors of altruism mainly focus on cognitive factors, such as moral identity, prosocial motivation and interpersonal trust, in addition, empathy also considered as an important effect factor. Empathy means that one has the ability to accurately share the feelings of others and understand the meaning of those feelings [2]. Baston proposes the Empathy-Altruism Hypothesis that when others are in trouble, bystanders will produce a kind of emotion pointing to the recipient, including empathy, sympathy, compassion, etc, and the greater the intensity of this emotion, the greater altruistic motivation of individuals to relieve others of their predicament, and then stimulate the occurrence of altruistic behavior [3]. And the supporters of the Empathy-Altruism Hypothesis believe that helping people can lead to a feeling of satisfaction. Furthermore, Hoffman put forward the threefold formula of empathy, moral principle and pro-social behavior, and hold that empathy as motivation directly leads to altruistic behavior, and empathy and moral principle work together for altruistic behavior [4]. Therefore, when individuals are more likely to feel the emotions and needs of others, they are more likely to generate altruistic behavior.

The previous researchers have verified the important effect of empathy on altruistic behavior through empirical studies. Some studies have found that inducing state empathy is an important motivator of helping behavior, and inducing empathy can promote the generation of altruistic behaviors, such as sharing behavior and donation behavior [5,6]. The influence of trait empathy and its components, which include emotional empathy, cognitive empathy, and empathic concern, have been found to be controversial in studies of altruism. Empathy was positively correlated with prosocial behavioral propensity and college students' altruistic behavior $[7,8]$, only emotional empathy could predict the networked altruistic behavior and the sharing behavior in the dictator game (DG) $[9,10]$, cognitive empathy alone could predict altruistic behavior in the ultimatum game(UG) [11]. However, other researchers hold that empathy only makes individuals feel the need 
for help from others, but it cannot determine the occurrence of altruistic behavior [12]. There are evidence to support this, some researchers found that trait empathy could not predicted the number of donations [6,13], a survey of 14 prosocial behaviors (including informal help by individuals and formal help through institutions) found that there was no significant correlation between empathic concerns and prosocial behaviors [14].

Combing the relevant literature found that the influence of empathy on altruism is not consistent, in addition, all of the above studies focused on explicit altruism. However, explicit altruistic behavior with high social approval, it is easy to be influenced by social judgment, self-cognition and expected future benefit. To address this question, the current study examined individual implicit altruism. Individual implicit altruism refers to the individual's and not necessarily conscious self-reward by helping others, which is an internalized behavior of social feedback, reflects the psychological level of the individual's inner consciousness but unwilling to report. And previous studies have shown that human altruism also exists on an unconscious level [15]. Implicit association test is usually used to measure individual's implicit altruism[16]. Therefore, this study used implicit association test to compare altruistic behavior of individuals with different empathy.

\section{EXPERIMENTAL METHODS}

\subsection{Selecting Subjects}

67 healthy Chinese college students come form Yunnan Normal University participated in this experiment, Delete the data whose accuracy rate in implicit association test is less than $80 \%, 58$ valid data were obtained. All subjects were healthy, right-handed, with normal or corrected to normal vision, and have no history of brain trauma, sleep disorders, etc.

\subsection{Experimental Design}

The mixed design of 2 ( empathy type: high group, low group) $\times 2$ ( task type: compatible task, incompatible task) was adopted, in which the empathy type was the between-subject variable, the task type was withinsubject variable, and the dependent variables included the accuracy, response time and IAT effect of implicit altruism test.

\subsection{Experimental Material}

\subsubsection{Empathy material.}

Chinese version of Interpersonal Reactivity Index (IRI-C) was used to measure empathy, and it revised by J.J. Wu, et al,which from the Interpersonal Reactivity Index compiled by Davis (1980) [17]. The questionnaire consists of 22 items, Likert 5 points were used in the questionnaire, From "totally inconsistent" to "completely consistent" score $1 \sim 5$, Cronbach's $\alpha$ is 0.68 in this study.

\subsubsection{Implicit altruism materials.}

Implicit association test was used to study altruistic behavior, The IAT consists of 20 words, which can be divided into two categories: target words and attribute words. Target words include altruistic and non-altruistic words; Attribute words include self and other words [16]. Altruistic words care, dedication, help, support, protection; non-altruistic words include rejection, attack, contempt, abuse, deception; Self words include oneself, individual, $\mathrm{i}$, we(all of us), we(both of us); other words include they(male), they(female), others, outsiders, another.

\subsection{Experimental Procedures}

When the subjects came to the laboratory, they asked to fill out demographic information and complete the Interpersonal Reactivity Index-C; and then asking subjects completed the implicit altruistic text according to the guidance.

E-Prime 1.1 software was used to write the computer experiment program of implicit altruism test. The stimulus presentation and related reactions were automatically recorded by the computer. The subject first read the instructions on the screen, and then press "SPACE" to start after clarifying the experiment requirements. At the beginning of each step, a description of the tasks and instructions for the phase are presented. During the test, labels ("self", "other","altruism" and "no-altruism") were presented on the upper left side and the upper right side of the screen, and stimulus vocabularies were presented in the center of the screen. If the subject classified the stimulus vocabularies into the upper left side, the "F" key was pressed. Press the "J" key if the stimulus word is in the upper right category. The IAT seven-step paradigm is adopted. The computer automatically records the time and error of each step. The specific steps and stimulus materials presented are shown in Table 1. 
Table1. Presentation materials for seven steps of the implicit association test

\begin{tabular}{|c|c|c|c|}
\hline Sequence & Task & Label & Vocabulary \\
\hline 1 & Initial target concept identification & altruism -no altruism & $\begin{array}{c}\text { altruistic-non altruistic } \\
\text { words }\end{array}$ \\
\hline 2 & $\begin{array}{c}\text { Associative target concept } \\
\text { identification }\end{array}$ & self - others & All words \\
\hline 3 & $\begin{array}{r}\text { Compatible task identification } \\
\text { (exercises) }\end{array}$ & self-altruism, others- no altruism & All words \\
\hline 4 & Compatible task identification (Formal) & self-altruism, others- no altruism & self - others \\
\hline 5 & $\begin{array}{r}\text { The opposite target concept } \\
\text { identification }\end{array}$ & self - others & All words \\
\hline 6 & $\begin{array}{r}\text { Incompatible task } \\
\text { identification(exercises) }\end{array}$ & others- altruism, self- no altruism & All words \\
\hline 7 & $\begin{array}{r}\text { Incompatible task } \\
\text { identification(Formal) }\end{array}$ & others- altruism, self- no altruism & ( \\
\hline
\end{tabular}

In this study, the response time and accuracy of compatible task in step 4 and incompatible task in step 7 were concerned. Compatible task refers to the relationship between concept words and attribute words consistent with the implicit attitude, that is, self-altruism. The incompatible task is the relationship between concept words and attribute words and the implicit attitude is inconsistent, that is, self-non-altruism.

\section{RESULT}

The response time is 3000 milliseconds if it is greater than 3000 milliseconds, 300 milliseconds if it is less than 300 milliseconds, with Interpersonal Reactivity Index score averages in boundary is divided into high empathy and low empathy group (high group of 29 people, low group 29) to data analysis.

\subsection{Operational inspection}

In order to test the grouping effectiveness of empathy, we conducted an independent sample t test, The scores of high empathy $(M=83.85, S D=4.83)$ were significantly higher than the low empathy group $(M=71.45, S D=5.74), \quad t(65)=9.58, p<0.001$, Cohen's $d=2.34$, indicating that the group of empathy was effective.

\subsection{Accuracy, Response time and IAT effect}

In order to investigate whether there are differences in accuracy between in two groups, we conducted a $2 \times$ 2 two-factor analysis of variance. Result is that the main effect of task type was significant, $F(1,56)=10.927, p$ $<0.01, \eta_{\mathrm{p}}^{2}=0.0163$, and the accuracy of compatible task is significantly higher than that of incompatible task; the main effect of empathy group was not significant, $F$ $(1,56)=0.392, p>0.05, \eta_{\mathrm{p}}^{2}=0.00$, the interaction between empathy type and task type was not significant, $F(1,56)=0.744, p>0.05, \eta_{\mathrm{p}}^{2}=0.013$.
On response time, we also conducted a $2 \times 2$ twofactor analysis of variance, it is found that the main effect of task type was significant, $F(1,56)=110.58, p$ $<0.001, \eta_{\mathrm{p}}^{2}=0.663$, the main effect of empathy group was not significant, $F(1,56)=0.016, p>0.05, \quad \eta_{\mathrm{p}}^{2}=$ 0.072 , but the interaction between empathy group and task type was significant, $F(1,56)=0.437, p<0.05$, $\eta_{\mathrm{p}}^{2}=0.001$. Further simple effect test was performed, in the low empathy group, the response time of compatible tasks was significantly lower than that of incompatible tasks, $t(28)=-5.90$, Cohen's $d=-1.55$, in the high empathy group, the response of compatible task was significantly lower than that of incompatible task, $t$ (28) $=-8.96$, Cohen's $d=-2.35$.

Table 2. Mean and standard deviation of response times(ms) for 58 subjects

\begin{tabular}{|c|c|c|}
\hline empathy type & compatible tasks & incompatible tasks \\
\hline low group & $899.34(45.27)$ & $1175.35(52.20)$ \\
\hline high group & $838.32(45.27)$ & $1251.73(52.20)$ \\
\hline
\end{tabular}

The IAT effect was obtained by subtracting response time of incompatible task from response time of compatible task. In order to investigate whether there are differences in the IAT effect between in two groups, we conducted a independent-samples $\mathrm{T}$ test. It is found that the IAT effect in the high empathy group was significantly greater than that in the low empathy group, $t(56)=2.074, p<0.05$, Cohen's $d=0.55$. 


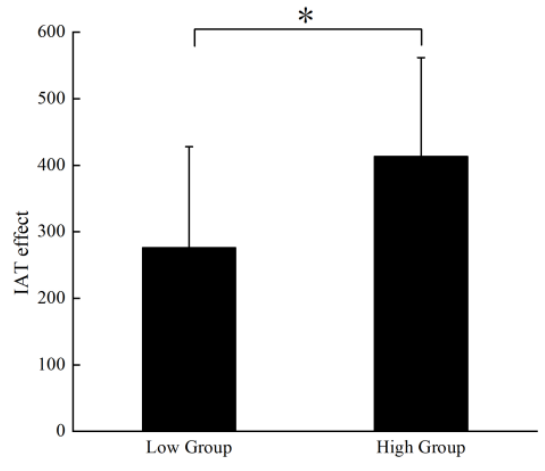

Fig1. IAT effect in different empathy groups

\section{DISCUSSION}

This study explored the effects of empathy on implicit altruism. Consistent with previous studies, compared with the incompatible tasks, the compatible task had higher accuracy and shorter response time [18]. In addition, the IAT effect of high empathy group was greater than that of low empathy group.

Consistent with previous research on implicit altruism, the accuracy of compatible tasks is higher than that of incompatible tasks, individuals agree more with altruistic related words, and the frequency of errors is lower. On the contrary, the frequency of errors is higher. Compatible task's response time is shorter than incompatible task, which proves the existence of IAT effect, and shows that altruistic behavior is also reflected in human unconscious level. In the process of individual socialization, self-concept and altruisticconcept form a connection network, which is easier to process, and the more automatic the individual considers altruistic tendency.

The IAT effect of high empathy group was greater than that of low empathy group, indicating that individuals with high empathy have more firm attitudes towards implicit altruism. High empathy individuals are more likely to feel the emotions and needs of others, experience a deeper socialization process, the more automatic altruistic tendency. Empathy-Altruism Hypothesis holds that when individuals are more likely to feel the emotions and needs of others, the more likely to produce altruistic motivation and behavior [3]. Another explanation is to alleviate their negative emotions. The Negative State Release Model holds that individuals can reduce their negative state by making prosocial behavior [19]. When the bystander sees that the victim is in trouble, he will also feel the negative emotion of the victim, and the individual will reduce his negative emotion by producing pro-social behavior. Therefore, the higher the empathy, the higher the altruism.

The experimental results can explain the emergence of explicit altruism. According to Social Information Processing Model, the early occurrence of individual altruistic behavior is the cue coding, that is, individuals notice the painful expressions of others for help and empathety to the pain of others [20]. Individuals with high empathy ability are more likely to have implicit altruistic tendency, and altruistic tendency stimulates altruistic behavior.

In school education, students' moral education is significance. Empathy can promote students' altruistic tendency, therefore, pay attention to empathy education to improve students' altruistic tendency and behavior. In future studies, the relationship between emotional empathy, cognitive empathy and implicit altruism can be subdivided, and then empathy intervention training can be conducted according to specific influencing factors to improve individuals' altruism tendency and behavior. This study provides certain supporting evidence for the promotion of altruism behavior by empathic training.

\section{CONCLUSION}

Empathy is related to implicit altruism. The higher the empathy, the higher the altruism. This study supports the Empathy-Altruistm Hypothesis.

\section{AUTHORS' CONTRIBUTIONS}

This research was completed with the joint efforts of four authors: X. Ma, Y Tao and D.N. Luo conducted the research; and D.N. Luo analyzed the data; D.N. Luo and T. Deng wrote the paper; all authors had approved the final version.

\section{ACKNOWLEDGMENTS}

We are grateful to all the participants for their contributions to the data collection, as well as Ms. Xu for revising the content, grammar, and format of the article.This study was supported by natural science foundation "cognitive control of ethnic bilinguals" (31660282) and the Yunnan Provincial Philosophy and Social Science Foundation "Innovative mechanism of minority middle school students' English syntax learning"(QN2017043).

\section{REFERENCES}

[1] B. Kerr, P.Godfrey-Smith, M.W. Feldman, What is altruism? Trends in Ecology \& Evolution, 2004, vol. 19(04).pp 135-144. DOI: 10.1016/j.tree.2003.10.004

[2] B.J. Kalisch, What is empathy? American Journal of Nursing, 1973,vol. 73(9), pp. 1548-1552.

[3] C.D. Batson, Empathy-Induced Altruistic Motivation. 2010. 
[4] M.L. Hoffman, Empathy and moral development. Cambridge university press, 2012, pp. 63-221. DOI: 10.5926/arepj1962.35.0_157

[5] J.A. Sze, G. Anett, M.S. Goodkind, \& R.W. Levenson. Greater emotional empathy and prosocial behavior in late life. Emotion, 2010, vol.12(5), pp.1129-40. DOI: 10.1037/a0025011

[6] O.M. Klimecki, S.V. Mayer, A. Jusyte, Empathy promotes altruistic behavior in economic interactions. Scientific Reports,2016, vol. 6, DOI: 31961.10.1038/srep31961

[7] L. Li, R.J. Zhang, Y.U. Ling, Y. Li, \&Y.M. Chen, Shyness and Prosocial Behavior among Junior Middle School Students: The Moderating Role of Empathy and its gender difference. Journal of Shandong Normal University( Natural Science), 2018,vol. 142(02), pp. 100-104.DOI: 10 . 3969 j. issn. 1001-4748. 2018. 02. 014

[8] W.H. Li, C. Li, Y. Shen \&F. Dan, Effect of Empathy on College Students' Altruistic Behavior: A Moderated Mediation Model. Psychological Development and Education, 2015, vol.(05), pp. 571-577. DOI: $\quad 10.16187 /$ j.cnki.issn10014918.2015.05.08

[9] Q. Zhao, Trait Empathy and Online Altruistic Behavior in College Students: Moderating Role of Moral Identity. Chinese Journal of Clinical Psychology, 2019,vol. (04). DOI: 10.16128/j.cnki.1005-3611.2019.04.033

[10] A. Edele, I. Dziobek, M. Keller, Explaining altruistic sharing in the dictator game: the role of affective empathy, cognitive empathy, and justice sensitivity. Learning and Individual Differences, 2013, vol. 24, pp. 96-102.DOI: 10.1016/j.lindif.2012.12.020

[11] Z. Li, J. Yu, X.Yang, \& L. Zhu, Associations between empathy and altruistic sharing behavior in chinese adults. The Journal of General Psychology, 2019, vol. 146(1), pp. 1-16.DOI: 10.1080/00221309.2018.1510826
[12] D.T. Kenrick, V. Griskevicius, J.M. Sundie, N.P. Li, \& S.L. Neuberg, Deep rationality: the evolutionary economics of decision making. Social Cognition, 2009,vol. 27(5), pp. 764. DOI: 10.1521/soco.2009.27.5.764

[13] R. Bekkers,Traditional and health-related philanthropy: the role of resources and personality. Social Psychology Quarterly, 2006, vol. 69. DOI: $10.1177 / 019027250606900404$

[14] C.J. Einolf, Empathic concern and prosocial behaviors: a test of experimental results using survey data. Social Science Research, 2008, vol. 37(4), pp. 1267-1279. DOI: 10.1016/j.ssresearch.2007.06.003

[15] D.G. Rand, J.D. Greene, M.A. Nowak, Spontaneous giving and calculated greed. Nature, 2012, vol. 489(7416), pp. 427-430. DOI: 10.1038 /nature1 1467

[16] A.G. Greenwald, D.E. Mcghee, J.L.K. Schwartz, Measuring individual differences in implicit cognition: the implicit association test. Journal of Personality \& Social Psychology, 1998, vol. 74(6), pp. 1464-80. DOI: 10.1037/0022-3514.74.6.1464

[17] M.H. Davis, A multidimensional approach to individual differences in empathy. Journal of Personality \& Social Psychology, 1980, vol. 10(85).

[18] D. Jiang, X.R. Wang, F. Li , R.L. Zhou, A Study on Implicit Altruistic Behavior. Psychological Science, 2008, vol.31(1), pp. 79-82. DOI:10.16719/j .cnki .1671 -6981.2008.01.017

[19] Y.Y. Guo, Personality Psychology-Research on Human Nature and Its Differences. Beijing: China Social Sciences Press, 2005, pp.438 -459.

[20] N.R. Crick, K.A. Dodge, A review and reformulation of social information-processing mechanisms in children's social adjustment. Psychological Bulletin, 1994, vol. 115(1), pp. 74101. DOI: $10.1037 / 0033-2909.115 .1 .74$ 